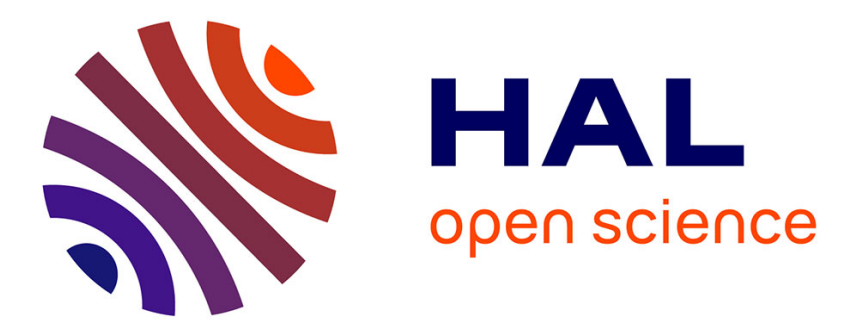

\title{
Anisotropie et cohérence spatiale du speckle ultrasonore dans les composites
}

\author{
A. Derode, M. Fink
}

\section{To cite this version:}

A. Derode, M. Fink. Anisotropie et cohérence spatiale du speckle ultrasonore dans les composites. Journal de Physique IV Proceedings, 1994, 04 (C5), pp.C5-1165-C5-1168. 10.1051/jp4:19945256 . jpa-00252945

\section{HAL Id: jpa-00252945 https://hal.science/jpa-00252945}

Submitted on 1 Jan 1994

HAL is a multi-disciplinary open access archive for the deposit and dissemination of scientific research documents, whether they are published or not. The documents may come from teaching and research institutions in France or abroad, or from public or private research centers.
L'archive ouverte pluridisciplinaire HAL, est destinée au dépôt et à la diffusion de documents scientifiques de niveau recherche, publiés ou non, émanant des établissements d'enseignement et de recherche français ou étrangers, des laboratoires publics ou privés. 


\section{Anisotropie et cohérence spatiale du speckle ultrasonore dans les composites}

\section{A. DERODE et M. FINK}

Laboratoire Ondes et Acoustique, ESPCI, Université Paris 7, URA 1503 du CNRS, 10 rue Vauquelin, 75005 Paris, France

Résumé : We briefly present experimental as well as theoretical results on measurements of spatial coherence of ultrasonic pulsed waves in random scattering media, particularly composite materials. We show that a given medium can behave either coherently or incoherently depending on the orientation of the ultrasonic beam, and what this implies in terms of characterization, speckle reduction and defect detection.

\section{Approche théorique générale}

Les techniques d'échographie ultrasonore utilisant des signaux temporels très brefs, il est possible de relier le temps d'arrivée d'un écho à la profondeur de la zone qui lui a donné naissance. Ainsi, en sélectionnant une fenêtre temporelle dans un signal échographique, on isole la contribution d'une tranche du matériau.

Cette approche permet de considérer la formation du signal échographique comme la réflection d'un signal incident sur un miroir de réflectivité aléatoire. Nous considérons alors indépendamment la propagation de chacune des fréquences constituant le signal incident. La théorie scalaire de la diffraction [1] nous permet d'écrire le champ dans le plan $\mathrm{z}$ comme :
$U_{z}(\vec{x})=O(\vec{X}) * e^{j \alpha \bar{X}^{2}}$
où $O(\vec{X})$ est la fonction d'ouverture du transducteur et $\alpha=\frac{\pi}{\lambda z}$

La réflection de cette onde sur le miroir se traduit par la multiplication par une variable aléatoire $\chi$ fonction de la position : $U_{z}^{r e ́ l}(\vec{x})=\chi(\vec{x}) U_{z}(\vec{x})$.

Ce champ réfléchi se propage alors jusqu'au transducteur, qui perçoit en chaque point $\vec{X}$ un champ rétrodiffusé :

$$
\psi(\vec{X})=U_{z}^{r e ́ f l}(\bar{x}) * e^{j \alpha \vec{x}^{2}}
$$

Cette expression contient une partie déterministe (la fonction d'ouverture, la fréquence, la profondeur $\mathrm{z}$ ) et une partie aléatoire (la réflectivité du milieu); il s'agit donc d'un signal aléatoire, dont on cherche à mesurer le moment d'ordre 2 , i.e. la fonction d'autocorrélation statistique définie par :

$$
R_{\psi \psi}\left(\vec{X}_{1}, \vec{X}_{2}\right)=E\left\{\psi\left(\vec{X}_{1}\right) \psi^{*}\left(\vec{X}_{2}\right)\right\}
$$

On cherche alors à relier la fonction de corrélation du signal rétrodiffusé à celle de la réflectivité aléatoire du milieu. Dans le cas d'un transducteur focalisé à la distance $F$, pour lequel on s'intéresse à une fenêtre temporelle autour du temps focal, on a pu montrer [2] la relation suivante:

$$
R_{\psi \psi}\left(\vec{X}_{1}, \vec{X}_{2}\right)=O\left(\vec{X}_{1}\right) O\left(\vec{X}_{2}\right)_{\vec{X}_{1} \vec{X}_{2}}^{*} \delta\left(\vec{X}_{1}-\vec{X}_{2}\right) T F\left\{R_{\chi \chi}\left(\vec{x}_{1}-\vec{x}_{2}\right)\right\}\left(\frac{\vec{X}_{1}}{\lambda F}\right)
$$

Cette fonction mesure le degré de corrélation statistique entre les champs perçus en $\bar{X}_{1}$ et $\vec{X}_{2}$ pour une fréquence donnée. Quand on s'intéresse à la cohérence spatiale d'un champ dans le domaine temporel, on est amené à calculer un degré de ressemblance entre des signaux $s(t)$ perçus en deux points $\vec{X}_{1}$ et $\vec{X}_{2}$ par :

$$
c\left(\vec{X}_{1}, \vec{X}_{2}\right)=\int s\left(\vec{X}_{1}, t\right) s\left(\vec{X}_{2}, t\right) d t
$$


On peut montrer [3] la relation entre ce degré de ressemblance et la fonction de corrélation statistique :

$$
E\left\{c\left(\vec{X}_{1}, \vec{X}_{2}\right)\right\}=\int R_{\psi \psi}\left(\vec{X}_{1}, \bar{X}_{2}\right) d \omega
$$

En moyenne, le degré de ressemblance mesuré dans le domaine temps est donc égal à la somme des fonctions de corrélation statistique sur tout le domaine fréquentiel exploré. Par la suite, nous nous intéresserons au cas d'un transducteur de forme rectangulaire, de dimensions axb, ce qui correspond au cas étudié expérimentalement (barrette linéaire).

\section{Milieu totalement incohérent}

Par abus de langage, on dit qu'un milieu est plus ou moins cohérent selon que la fonction $R_{\chi \chi}\left(\vec{x}_{1}-\vec{x}_{2}\right)$ sera large ou étroite. Pour un milieu totalement incohérent, on a $R_{\chi \chi}\left(\vec{x}_{1}-\bar{x}_{2}\right) \propto \delta\left(\vec{x}_{1}-\vec{x}_{2}\right)$. Dans ce cas, on obtient simplement :

$$
R_{\psi \psi}\left(X_{1}, X_{2}\right)=O(X) \underset{\Delta X}{*} O(X)=\operatorname{tri}\left(\frac{\Delta X}{a}\right)=1-\frac{|\Delta X|}{a} \text { pour } \quad|\Delta X|<a
$$

\section{Milieu parfaitement cohérent}

Un milieu parfaitement cohérent se comporte comme un miroir parfaitement lisse, on a $R_{\chi \chi}\left(\bar{x}_{1}-\vec{x}_{2}\right)=$ cste et on obtient : $\quad R_{\psi \psi}\left(X_{1}, X_{2}\right)=O\left(X_{1}\right) O\left(X_{2}\right)=\operatorname{rect}\left(\frac{X_{1}}{a}\right) \operatorname{rect}\left(\frac{X_{2}}{a}\right)$

Ceci signifie que pour un milieu parfaitement cohérent, sur toute l'étendue de l'ouverture émettrice, les signaux reçus autour du temps focal seront parfaitement identiques; alors que dans le cas d'un milieu complètement incohérent, le degré de ressemblance entre les signaux captés en deux points chute linéairement avec la distance séparant ces deux points, jusqu'à ce que cette distance soit égale à la largeur de l'ouverture en émission, auquel cas il n'y a plus aucune ressemblance.

Le cas d'un milieu à cohérence partielle, c'est-à-dire présentant une longueur de cohérence ni nulle ni infinie peut être traité de la même manière. On peut ainsi montrer que l'on obtient une forme intermédiaire entre le triangle et le rectangle, selon la largeur spectrale des signaux.

\section{Cas des milieux composites}

Un composite unidirectionnel est formé de très longues et très fines $(7 \mu)$ fibres de carbone noyées dans une matrice de résine, alignées selon une même direction. Tout se passe donc comme si le milieu était déterministe dans la direction d'alignement des fibres et complètement aléatoire dans les directions perpendiculaires. On modélise ainsi la fonction d'autocorrélation de la réflectivité par :

$$
R_{\chi \chi}\left(\vec{x}_{1}-\vec{x}_{2}\right) \propto \delta\left(x_{1}-x_{2}\right) \text {, si les fibres sont alignées selon l'axe y. (Fig. 1) }
$$

On conçoit que les propriétés de cohérence spatiale du champ acoustique rétrodiffusé par un tel matériau vont profondément changer selon la direction des fibres par rapport à l'axe de la barrette. On peut effectivement montrer que lorsque la direction d'alignement est parallèle à l'axe de la barrette, le milieu se comporte comme un milieu parfaitement cohérent (degré de ressemblance constant); alors que lorsque la barrette est perpendiculaire à l'axe des fibres, le milieu se comporte de façon incohérente (degré de ressemblance décroissant comme un triangle). Pour les angles intermédiaires on observe une transition entre cohérence et incohérence, la transition étant gouvernée par le rapport $b / a$, ou $b$ est la hauteur de chaque élément de la barrette et a la longueur de l'ouverture active en émission.

\section{Méthode et résultats expérimentaux}

Le dispositif expérimental est schématisé sur la figure $1:$ la barrette est fixée sur un dispositif rotatif qui permet de fixer son orientation par rapport à la direction d'alignement des fibres. Elle comporte 128 éléments de pas $426 \mu$; la focalisation est naturelle, car la barrette a un rayon de courbure de $90 \mathrm{~mm}$. Dans un premier temps, lopérateur choisit la taille de la surface active à l'émission (on peut ne faire tirer que 16 ou 32 éléments par exemple); le signal d'émission est constitué de 2 périodes de sinusoïde à $3,5 \mathrm{MHz}$ (fréquence centrale de la barrette). Les 128 voies de la barrette enregistrent alors un bruit rétrodiffusé par le milieu. Pour chacun de ces 128 signaux, on sélectionne une fenêtre temporelle $\left[T_{1}, T_{2}\right]$ centrée sur le temps focal, et l'on corrèle les signaux deux à deux : 


$$
\rho(i, j)=\sum_{T_{1}}^{T_{2}}\left(s_{i}(t)-\bar{s}_{i}\right)\left(s_{j}(t)-\bar{s}_{j}\right) \quad \text { avec } \quad \bar{s}_{k}=\frac{1}{T_{2}-T_{1}} \sum_{T_{1}}^{T_{2}} s_{k}(t)
$$

Puis on moyenne $\rho$ pour tous les couples de capteurs espacés de la même distance de façon à obtenir une estimation du degré de ressemblance en fonction de la distance :

$$
c(m)=\frac{N}{N-m} \sum_{i=1}^{N-m} \rho(i, i+m) / \sum_{i=1}^{N} \rho(i, i)
$$

Les courbes expérimentales représentant $\mathrm{c}(\mathrm{m})$ pour le composite unidirectionnel sont représentées sur la Fig. 2. On observe ainsi que dans le cas de l'orientation perpendiculaire, le degré de ressemblance est très sensible à la taille de l'ouverture active en émission : plus la source est petite, plus le degré de ressemblance chute brutalement. En revanche, dans le cas de l'orientation parallèle, les signaux captés en différents points de la barrette conservent une très forte ressemblance. Cela est également frappant sur les signaux présentés sur la figure 3.

\section{Composite à fibrage complexe}

La même approche tant expérimentalement que théoriquement à été appliquée à des composites bi- et quadri-directionnels, qui sont formés de couches élémentaires de fibres empilées avec un pas hélicoïdal respectif de $90^{\circ}$ et $45^{\circ}$. On peut ainsi mettre en évidence dans le signal plusieurs contributions -cohérente, incohérente ou partiellement cohérente- provenant de couches élémentaires différemment orientées parallèle, perpendiculaire, intermédiaire- par rapport à la barrette.

Selon l'importance respective de ces contributions, le signal total est soit plutôt cohérent, soit plutôt incohérent, selon la valeur du paramètre b/a. Avec les dimensions de notre barrette, il semblerait que le caractère incohérent l'emporte sur un composite bidirectionnel, alors que pour le composite quadridirectionnel, le signal rétrodiffusé est très cohérent (Fig. 4).

\section{Conclusion}

Connaitre les propriétés de cohérence spatiale du bruit diffusé par un milieu donné est fondamental à plusieurs égards : comme nous l'avons vu, cela permet de remonter à une information sur la statistique des diffuseurs; d'autre part, plus le bruit de speckle est cohérent plus il risque de masquer l'écho d'un réflecteur important (défaut, lésion); enfin, en connaisant bien les propriétés de cohérence du bruit diffusé par la structure d'un milieu 'sain', on peut espérer identifier l'écho d'un réflecteur important (défaut,lésion) par sa nature spatialement cohérente et non par sa seule amplitude.

\section{Réferences}

[1] Athanasios PAPOULIS, Systems and transforms with applications in optics, p. 366, Mc Graw-Hill, 1968.

[2] Arnaud DERODE, Mathias FINK, "Spatial coherence of ultrasonic speckle noise in composites", IEEE Transactions on Ultrasonics, Ferroelectrics and Frequency Control, nov. 1993.

[3] Arnaud DERODE, Mathias FINK, "The notion of coherence in optics and its application to acoustics", European Journal of Physics, jan. 1994.

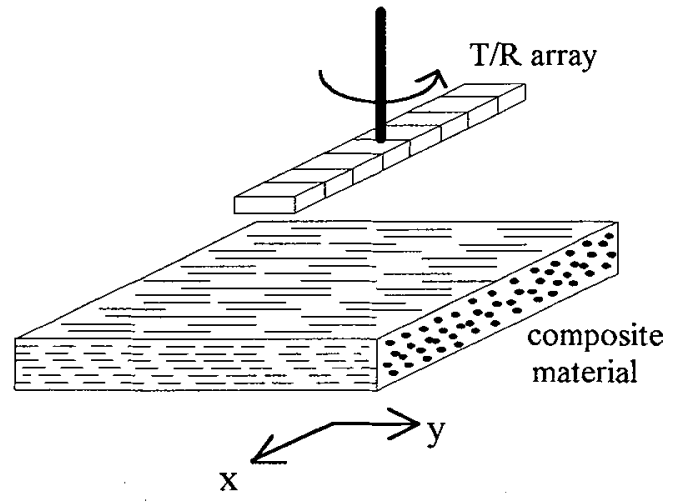

Fig. 1 : Dispositif expérimental 


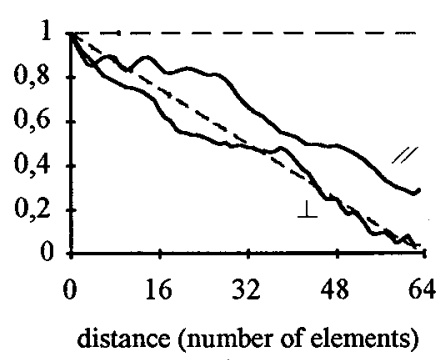

(a)

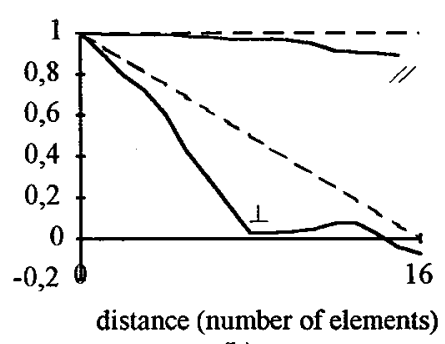

(b)

Fig. 2 : Coefficient de corrélation $c(m)$ en fonction de la distance. Tirets : résultats théoriques. (a) : orientations perpendiculaire $(\perp)$ et parallèle $(/ /)$, ouverture à l'émission : 64 éléments (b) : orientations perpendiculaire $(\perp)$ et parallèle ( $(/)$, ouverture à l'émission : 16 éléments

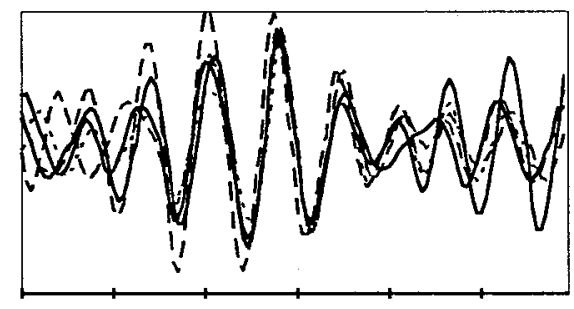

(a)

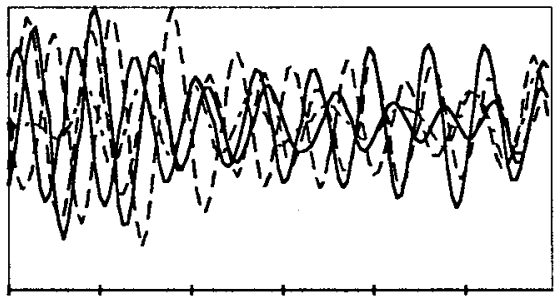

(b)

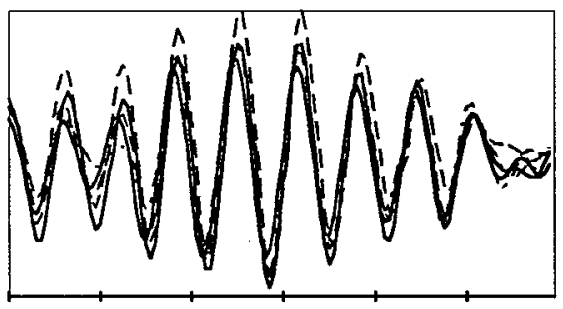

(c)

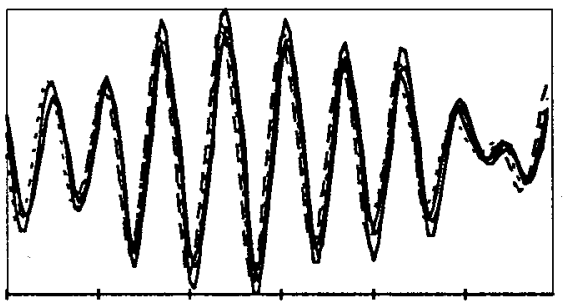

(d)

Fig. 3 : 5 signaux temporels reçus sur 5 éléments de la barrette dans 4 cas différents :

(a) : orientation perpendiculaire, ouverture à l'émission : 64 éléments

(b) : orientation perpendiculaire, ouverture à l'émission : 16 éléments

(c) : orientation parallèle, ouverture à l'émission : 64 éléments

(d) : orientation parallèle, ouverture à l'émission : 16 éléments

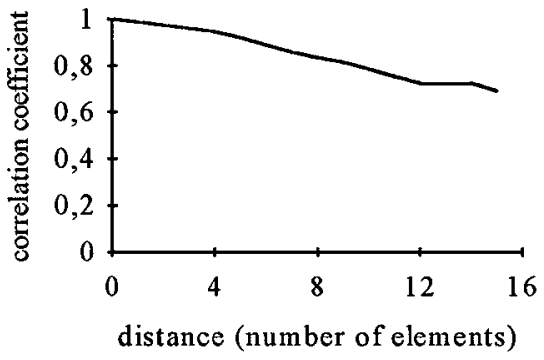

(a)

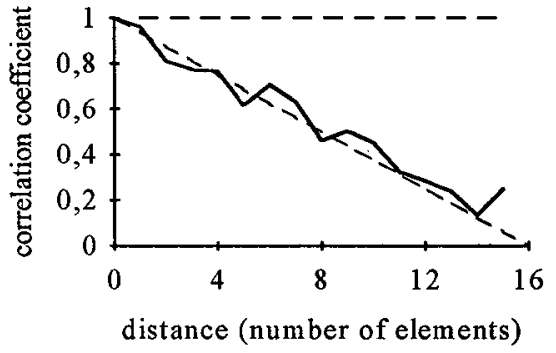

(b)

Fig. 4 : Coefficient de corrélation $c(m)$ en fonction de la distance. Ouverture à l'émission : l6 éléments (a) composite quadridirectionnel (b) composite bidirectionnel 